\title{
Measuring fusion excitation functions with RIBs \\ using the stacked target technique: Problems and possible solutions
}

\author{
M. Fisichella ${ }^{1}$, A. C. Shotter ${ }^{2}$, A. Di Pietro $^{1}$, P. Figuera $^{1}$, \\ M. Lattuada ${ }^{1,3}$, C. Marchetta ${ }^{1}$, V. Privitera ${ }^{4}$, L. Romano $^{3,4}$, \\ C. RuIZ ${ }^{5}$ and M. ZADRO 6
}

${ }^{1}$ INFN - Laboratori Nazionali del Sud, via S. Sofia 62, I-95123 Catania, Italy

${ }^{2}$ School of Physics and Astronomy, University of Edinburgh, JCMB, Mayfield Road, Edinburgh EH9 3JZ, UK

${ }^{3}$ Dipartimento di Fisica ed Astronomia, via S. Sofia 64, I-95125 Catania, Italy ${ }^{4}$ CNR-IMM MATIS, via S. Sofia 64, Catania, Italy ${ }^{5}$ TRIUMF, 4004 Wesbrook Mall, Vancouver, BC V6T 2A3, Canada ${ }^{6}$ Ruđer Bošković Institute, Bijenička cesta 54, HR-10000 Zagreb, Croatia

\begin{abstract}
For measuring fusion excitation functions, the activation technique with a stack of targets offers the considerable advantage that several reaction energies may be simultaneously measured by using one beam energy. However, its main drawback is the degradation of the beam quality as it passes through the stack due to statistical nature of energy loss processes and any non-uniformity of the stacked targets. If not taken properly into account, this degradation can lead to a wrong determination of the fusion excitation function. In this contribution some results of the investigation of this problem are reported.
\end{abstract}




\section{Introduction}

In the last decades, many fusion reactions induced by low intensity RIBs have been studied by irradiating stacks of several thick targets and measuring on-line or off-line the radiation emitted in the decay of the evaporation residues $[1-7]$.

In these activation experiments, the fusion excitation function has been determined by associating the cross-section $\sigma_{\text {mean }}(\mathrm{E})$, measured in each target, either with the energy of the beam in the middle of the considered target $\bar{E}$ or to the effective energy $E_{\text {eff }}$, defined as follows:

$$
E_{e f f}=\frac{\int_{0}^{\infty} E \sigma(E) D\left(E, t_{0}\right) d E}{\int_{0}^{\infty} \sigma(E) D\left(E, t_{0}\right) d E}
$$

In the last definition, the value of the energy is obtained by an averaging procedure which weights the beam energy with energy distribution $D\left(E, t_{0}\right)$ inside the considered target of thickness $t_{0}$ together with the trend of the fusion cross sections. The $D\left(E, t_{0}\right)$ distribution represents the probability that a beam particle will have energy $E$ inside the considered target of thickness $t_{0}$. The physical factors that influence the shape of the energy distribution function $D\left(E, t_{0}\right)$ are the shape of the beam energy distribution entering the target, the beam energy loss in the target, and the energy straggling of the beam. The $D\left(E, t_{0}\right)$ distribution taking esplicitly into account all the above effects, can be determined by using the SRIM code [9] as discussed in [8].

The present authors found that neither $\bar{E}$ nor $E_{\text {eff }}$ lead to the correct reconstruction of the fusion excitation function. In particular, it has been found that in the case of an uniform target $\bar{E}$ is an underestimation and $E_{\text {eff }}$ is an overestimation, respectively, of the real value $E_{r}$, defined as the reaction energy such that $\sigma_{\text {mean }}=\sigma\left(E_{r}\right)$, where $\sigma(E)$ is the true cross section function. As an example it was supposed to know the behaviour of the ${ }^{9} \mathrm{Li}+{ }^{120} \mathrm{Sn}$ fusion excitation function and it was supposed that at subbarrier energies it has an exponential trend with a slope $\alpha=1.72 \mathrm{MeV}^{-1}$, which is within the range of typical slopes observed in sub-barrier fusion experimental data. We considered an incoming energy beam of $17 \mathrm{MeV}$ and calculated analitically the values of $E_{r}, \bar{E}$ and $E_{\text {eff }}$ as a function of the energy losses in the target (i.e., targets of different thicknesses). The results are reported in figure 1 in the case of a delta incoming beam energy distribution (Fig.1a) and Gaussian incoming beam energy distribution with FWHM $=2 \mathrm{MeV}$ (Fig.1b). Only for the ideal case of a mono-energetic beam and for thin targets will $E_{r}, \bar{E}$, and $E_{e f f}$ coincide. In this example uniform 

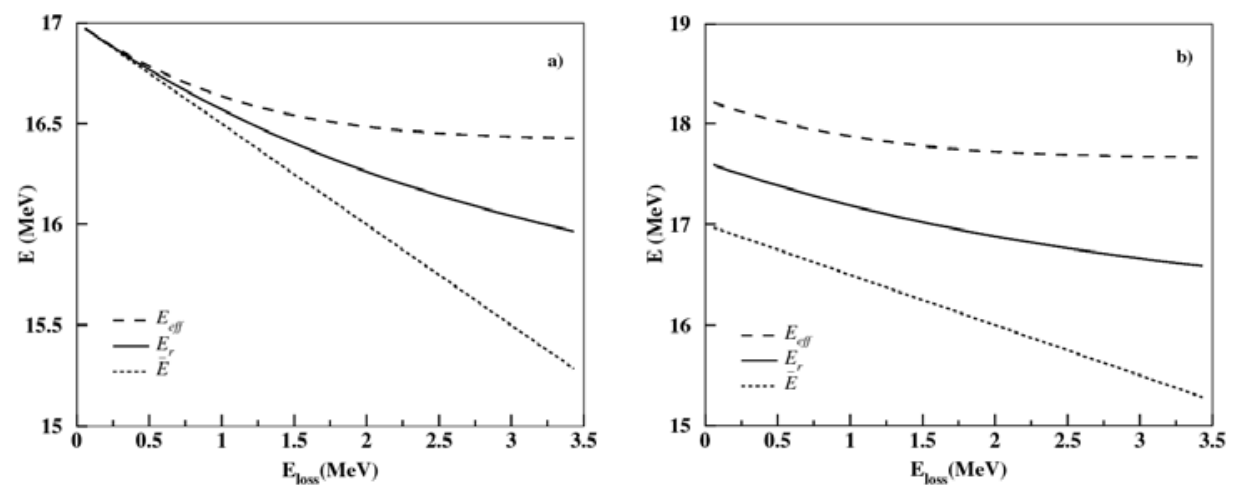

Figure 1: Calculations for the ${ }^{9} \mathrm{Li}+{ }^{120} \mathrm{Sn}$ system. $\bar{E}, E_{\text {eff }}$ and $E_{r}$ are plotted as a function of the energy loss in the target for an average incident beam energy is $17 \mathrm{MeV}$. a) Delta beam energy distribution; b) Gaussian beam energy distribution with $\mathrm{FWHM}=2 \mathrm{MeV}$. See text for details.

targets have been considered, but it has to be noticed that the presence of non-uniformities in the target will further modify this behaviour, as it will be shown in the following section.

The present authors developed an alternative deconvolution procedure to determine the correct fusion excitation function of the studied system. The idea is to determine an analytical expression for $\sigma(\mathrm{E})$ such that for the $D\left(E, t_{0}\right)$ associated to each given target one would obtaion by using Eq. 3 the measured mean cross section. Details about this new method are reported in [8]. Application of this procedure requires a detailed knowledge of $D\left(E, t_{0}\right)$. The best way to determine $D\left(E, t_{0}\right)$ is to characterise target properties, as well as performing numerical beam energy loss simulations. Of course, these effects are amplified when a stack of targets is used. For this reason, an accurate analysis has been done concerning the the target non-uniformity and the effect that this could have on the determination the fusion excitation function. It has been developed an experimental technique based on simple measurements of residual energy spectra of $\alpha$ emitted by an ${ }^{231} \mathrm{Am}$ source from which it is possible to determine in a very accurate way the target thickness distribution [8].

\section{$2{ }^{9} \mathbf{L i}+{ }^{120} \mathrm{Sn}$ simulation}

Generally, in the previous articles where these kind of experiments are discussed, very few information are given about target non-uniformity, straggling effects and incident beam quality. However, as discussed previously, 
since these factors can significantly influence the deduced excitation functions the present authors performed simulations in order to investigate in details how they might influence the determination of the fusion excitation function and try to quantify the magnitudes of these effects.

Numerical simulations of the ${ }^{9} \mathrm{Li}+{ }^{120} \mathrm{Sn}$ fusion reaction have been performed. It has been supposed that a $28 \mathrm{MeV}{ }^{9} \mathrm{Li}$ beam impinged on a stack of five targets each composed of one ${ }^{120} \mathrm{Sn}$ foil with an average thickness of $5 \mathrm{mg} / \mathrm{cm}^{2}$, followed by an uniform ${ }^{93} \mathrm{Nb}$ catcher/degrader foil $1.5 \mathrm{mg} / \mathrm{cm}^{2}$ thick. The results of the simulations were studied for the case of uniform targets and degrader foils, and for the situation where both targets and foils are non-uniform. The ${ }^{9} \mathrm{Li}+{ }^{120} \mathrm{Sn}$ fusion excitation function behaviour has been supposed to be known and to have a Wong-like behaviour:

$$
\sigma(E)=\frac{A}{E} \ln \left(1+e^{B(E-C)}\right)
$$

with $\mathrm{A}=1936 \mathrm{mb} \cdot \mathrm{MeV}, \mathrm{B}=1.83 \mathrm{MeV}^{-1}, \mathrm{C}=18.95 \mathrm{MeV}$. The chosen parameters in the Eq.(2) are consistent with the cross-section slopes of other similar reactions. In this way, for each target, the mean cross-section that would be measured in it has been calculated by using the following relation:

$$
\sigma_{\text {mean }}=\frac{\int_{0}^{\infty} \sigma(E) D\left(E, t_{0}\right) d E}{\int_{0}^{\infty} D\left(E, t_{0}\right) d E}
$$

As an example, in Fig. 2a the $D\left(E, t_{0}\right)$ distributions of the fitht target obtained by the SRIM simulation for the case of uniform (continuos line) and non-uniform (dotted line) stack foils are reported. As it is possible to see, not only the shapes but also the explored energy ranges of the distributions are different in the two cases.

In Fig. $2 \mathrm{~b}$ the $\sigma_{\text {mean }}$ obtained by applying Eq. (3) for the uniform and nonuniform target cases, is plotted versus the mean energy $\bar{E}$ (open symbols), and versus the effective energy $E_{\text {eff }}$ (closed symbols). The circles and triangles correspond to case of uniform and non-uniform target, respectively. It has to be put in evidence that the cross-section values that would be measured for the two cases are slightly different due to the different energy distributions inside the targets. The continuous line represents the cross-section of Eq. (2) assumed to represent the real behavior of the fusion excitation function in the simulated experiment. As one can see, at energies below the Coulomb barrier, the real excitation function is not reproduced by any of the two representations. The differences are bigger in the case of non-uniform targets with respect to the uniform case, as one can expect due to the wider energy distributions $D\left(E, t_{0}\right)$. Plotting the measured 

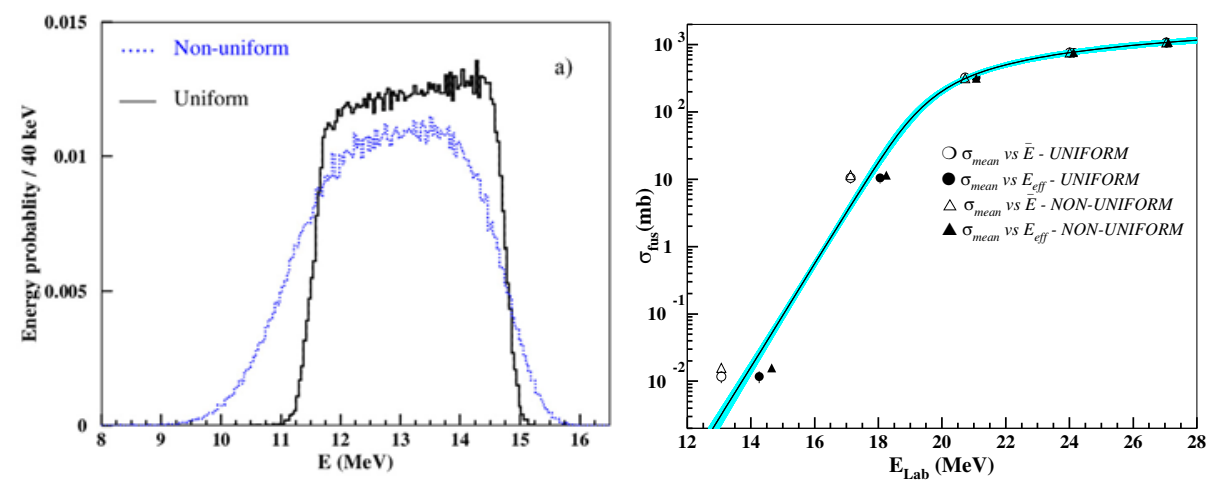

Figure 2: a) Energy distribution of the beam inside the fifth target of the stack considering uniform (continuous line), and non uniform (dotted line) foils. b) Results of the simulations for the case of uniform (circles) and for non-uniform (triangles) targets. The continuous black line shows the real fusion excitation function assumed for the collision ${ }^{9} \mathrm{Li}+{ }^{120} \mathrm{Sn}$. The open and closed symbols are the experimental results that would be obtained plotting the measured $\sigma_{\text {mean }}$ versus $\bar{E}$ and $E_{\text {eff }}$. In this figure the results of the deconvolution procedure cannot be distinguished from the black line. The blue shaded area represent the uncertainty associated with the deconvolution procedure. See text for details.

cross-sections versus $\bar{E}$ or $E_{\text {eff }}$ generates differences up to a factor of 5 with respect to the real fusion excitation function.

The deconvolution procedure, developed by the present authors, has also been applied to these simulated data. For applying this procedure a typical error on the measured mean cross-section, $\sigma_{\text {mean }}$, in each of the targets, has been supposed to be $15 \%$. This error would originate from experimental uncertainties associated with detector efficiencies, mean target thickness, target thickness distribution etc. The results obtained with this procedure cannot be distinguished from the continuum line representing the assumed real cross-section. The shaded blue area represents the error band associated with the procedure. This error band has been calculated by applying the standard error propagation formula to Eq. 2, considering the three parameters $(\mathrm{A}, \mathrm{B}, \mathrm{C})$ as free variables.

According to the present results the best way of determining the fusion excitation function is to use the deconvolution procedure. 


\section{Summary and conclusion}

From the present study it is clear that that the traditional way to represent the fusion cross section as function of the energy in the middle of the target, or as a function of an effective energy based on a weighted average which takes into account both the beam energy distribution and the energy dependence of the cross section, may lead to a wrong determination of the fusion excitation function, especially when the energy distribution within a target is large, and the cross-section is rapidly changing with the energy. The present authors suggest as a possible solution to infer the true excitation function, to apply a deconvolution procedure as discussed in previous paragraph. In order properly apply this procedure a detailed knowledge of the the target thickness distribution and of the incoming beam distribution beam must be available. Therefore the authors recommend that for future publications where such data is presented, enough information should be included concerning the used foil non-uniformities, beam energy distribution and their effects on the deduced fusion excitation functions.

\section{References}

[1] J. J. Kolata, et al., Phys. Rev. Lett. 81, 4580 (1998).

[2] P. A. DeYoung, et al, Phys. Rev. C 58, 34423444 (1998).

[3] Y. Penionzhkevich, et al., The European Physical Journal A 31, 185194 (2007).

[4] A. Lemasson, et al., Phys. Rev. Lett. 103, 232701 (2009).

[5] Y. E. Penionzhkevich, et al, Phys. Rev. Lett. 96, 162701 (2006).

[6] C. Signorini, et al, Nuclear Physics A 735, 329344 (2004).

[7] R. Wolski, et al., The European Physical Journal A 47, 111 (2011).

[8] M. Fisichella et al., Accepted for publication in Phys. Rev. C

[9] J. Ziegler, Srim code - http://www.srim.org .

[10] C. Y. Wong, Phys. Rev. Lett. 31, 766769 (1973). 